\title{
Radiofrequency heat-enhanced direct intratumoral chemotherapy for prostate cancer
}

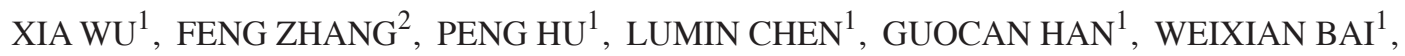 \\ JINGFENG LUO ${ }^{1}$, RAN CHEN $^{3}$, YURONG ZHOU ${ }^{1}$, JIHONG SUN $^{1}$ and XIAOMING YANG ${ }^{1,2}$ \\ ${ }^{1}$ Department of Radiology, Sir Run Run Shaw Hospital, Zhejiang University College of Medicine \\ and Sir Run Run Shaw Institute of Clinical Medicine of Zhejiang University, Hangzhou, Zhejiang 310016, P.R. China; \\ ${ }^{2}$ Image-Guided Bio-Molecular Intervention Research and Section of Vascular and Interventional Radiology, \\ Department of Radiology, University of Washington School of Medicine, Seattle, WA 98195, USA; ${ }^{3}$ Department of \\ Diagnostic Ultrasound and Echocardiography, Sir Run Run Shaw Hospital, Zhejiang University College of Medicine \\ and Sir Run Run Shaw Institute of Clinical Medicine of Zhejiang University, Hangzhou, Zhejiang 310016, P.R. China
}

Received January 21, 2016; Accepted June 9, 2017

DOI: 10.3892/ol.2017.7145

\begin{abstract}
A novel, minimally invasive interventional technique, radiofrequency heat $(\mathrm{RFH})$, has been suggested to improve the efficacy of chemotherapy for solid organ tumors. However, the treatment for prostate cancer has not been completely characterized. The aim of the present study was to investigate the in vitro and in vivo efficiency of chemotherapy in combination with RFH for the treatment of prostate cancer. The following four treatment groups were included: i) No treatment (control); ii) RFH-only; iii) chemotherapy (docetaxel)-only; and iv) combination therapy of docetaxel and RFH in human prostate cancer (HPC) cell lines and mice with HPC xenografts. In the in vitro experiments, a heating guidewire was attached under the bottom of the last chamber of the four-chamber cell culture slide, and was then connected to a radiofrequency (RF) generator. In the in vivo experiments, a tumor model was generated by subcutaneously injecting human prostate cancer cells into 24 male nu/nu mice. $\mathrm{RFH}$ was conducted by inserting the 0.022 -inch heating-guidewire into the tumor. The follow-up magnetic resonance imaging demonstrated a significant reduction in the average tumor size in animals treated with combination therapy compared with those receiving RFH-only and chemotherapy-only. The number of apoptotic cells and the average apoptotic index of the combination therapy group were significantly higher
\end{abstract}

Correspondence to: Dr Xiaoming Yang, Image-Guided Bio-Molecular Intervention Research and Section of Vascular \& Interventional Radiology, Department of Radiology, University of Washington School of Medicine, 850 Republican Street, S470, Seattle, WA 98195, USA

E-mail: xmyang@u.washington.edu

Key words: prostate cancer, radiofrequency, docetaxel, magnetic resonance imaging, hyperthermia compared with those of the other three treatment groups. In conclusion, the results of the present study suggested that $\mathrm{RFH}$ is able to increase the therapeutic efficiency of docetaxel in prostate cancer, and this study serves as a foundation for the future development of an interventional molecular image-guided local treatment strategy for prostate cancer that integrates RF technology, interventional oncology and direct intratumoral chemotherapy, as a replacement for systemic chemotherapy.

\section{Introduction}

Prostate cancer (PC) is the second most frequently diagnosed type of cancer and the sixth leading cause of cancer mortality worldwide (1,2). An estimated 160,000 new cases of prostate cancer were diagnosed annually in the United States (3). With the widespread use of prostate-specific antigen (PSA) screening and prostate biopsy, an increased number of men are being diagnosed with low-grade and localized prostate cancer, and a large portion of prostate cancer cases are being diagnosed as unifocal and unilateral lesions (4). Overtreatment has occurred in $>30 \%$ of these patients (5). Traditional whole-gland radical therapies, including external beam radiotherapy and radical prostatectomy (RP), cannot achieve the local removal of lesions without damaging adjacent normal structures, such as the urethra and colorectum (5).

Docetaxel is one of the most potent chemotherapy drugs used for the treatment of prostate cancer (6). However, its clinical applications via systemic delivery are limited by its low chemotherapeutic distribution at the tumor site and its high toxicity to other vital organs (7). Furthermore, previous studies also suggested that an insufficient deposition of chemotherapeutic drugs at the target tumor via systemic administration contributes significantly to the development of cancer chemoresistance (8). Therefore, a local approach to administration to increase the accumulation of the chemotherapeutics at the target lesions and decrease the accumulation in other areas, such as by direct intratumoral administration 
of chemotherapeutics via interventional oncology techniques, may resolve this issue. In addition, hyperthermia with a temperature of $\sim 42^{\circ} \mathrm{C}$ may increase the effectiveness of chemotherapy in a variety of malignancies (9-11). However, the lack of adequate devices for local heat delivery to the targets and appropriate temperature monitoring methods at the target sites, limits the application of hyperthermia-enhanced therapy for prostate malignancies (12).

Therefore, an image-guided, minimally invasive interventional approach that not only permits the local delivery of highly concentrated therapeutics to the target site, but also avoids therapeutic toxicity to other vital organs (which is often a consequence of systemic delivery approaches) should be investigated (13). Although previous advancements in interventional oncology technologies have improved the outcomes of patients with cancer (7), the present study hypothesized that the combination of interventional oncology and local hyperthermia may additionally improve the efficacy of chemotherapies in organ cancer. Therefore, a novel technique interventional radiofrequency heat (RFH), which combines these two aforementioned conditions, was considered. Previous studies by the authors have demonstrated an improvement in efficacy of chemotherapy in cholangiocarcinoma and breast cancer by RHF $(10,14)$. Therefore, the aim of the present study was to identify whether the efficiency of direct intratumoral chemotherapy for prostate cancer may be enhanced using this novel method, RHF.

\section{Materials and methods}

The present study was divided into two phases: i) In vitro 'proof-of-principle' experiments which demonstrate that RFH enhances chemotherapeutic efficiency in human prostate cancer cells; and ii) in vivo validation that interventional RFH enhances direct intratumoral chemotherapy for prostate cancer in a xenograft animal model.

Cell lines. The human prostate cancer PC-3 cell line (American Type Culture Collection, Manassas, VA, USA) was used. The PC-3 cell line was initiated from a bone metastasis of a grade IV prostatic adenocarcinoma from a 62-year-old male Caucasian. The PC-3 cell line was cultured in RPMI-1640 media (Gibco; Thermo Fisher Scientific, Inc., Waltham, MA, USA) supplemented with $10 \%$ fetal bovine serum (Gibco; Thermo Fisher Scientific, Inc.) at $37^{\circ} \mathrm{C}$ in a humidified incubator with a $5 \%$ $\mathrm{CO}_{2}$ atmosphere.

In vitro experimental model. Each chamber of a Lab-Tek ${ }^{\circledR}$ 4-chamber cell culture slide ${ }^{\mathrm{TM}}$ (Thermo Fisher Scientific, Inc.) was seeded with $2 \times 10^{4} \mathrm{PC}-3$ cells. The slide was placed in a water bath at $37^{\circ} \mathrm{C}$. A 0.032 inch heating guidewire was attached under the bottom of chamber 4 of the four-chamber cell culture slide and was then connected to a radiofrequency (RF) generator (MPG-4; OPTHOS Instruments, Inc., Rockville, MD, USA) When the RF generator was operated at 2-3 W through the heating guidewire, the temperature in chamber 4 increased from $37-42^{\circ} \mathrm{C}$, creating a heat gradient along the four chambers (Fig. 1). The temperature of each chamber was recorded using a micro-thermometer (Photon Control Inc., Richmond, Canada).
To compare the therapeutic effects of different RFH temperatures, the same amount of docetaxel (52 nM) (Hopira, Lake forest, IL, USA) was placed into each of the four chambers as the bottom of chamber 4 was heated to $42^{\circ} \mathrm{C}$ for $20 \mathrm{~min}$, and the temperature of chamber 1 was maintained at $37^{\circ} \mathrm{C}$. To compare the therapeutic effects of different treatments, the prostate cancer cells were divided into four groups: No treatment (control), RFH only $\left(42^{\circ} \mathrm{C}\right.$ for $\left.20 \mathrm{~min}\right)$, chemotherapy only (chemo-only, $52 \mathrm{nM}$ docetaxel) and combination therapy (chemotherapy plus RFH). A docetaxel concentration of $52 \mathrm{nM}$ was selected according to previous studies $(15,16)$. Following this, the cells were then cultured at $37^{\circ} \mathrm{C}$ for $72 \mathrm{~h}$, and subjected to different laboratory evaluations, including cell proliferation and apoptosis assays, to examine and compare the effects of the different treatments on prostate cancer cells.

The treatments were initiated when the cell confluency reached $80 \%$. RFH-enhanced chemotherapy was performed by adding docetaxel into the medium and heating the cells to $\sim 42^{\circ} \mathrm{C}$ with the RF power set to $12-14 \mathrm{~W}$ for $20 \mathrm{~min}$. Docetaxel was maintained in the chamber for $24 \mathrm{~h}$ following RFH. The cells in the chemotherapy-only group were treated with docetaxel for the same duration $(24 \mathrm{~h})$.

Cell proliferation assay. Cell proliferation was evaluated by the MTS assay (Promega Corp., Madison, WI, USA). Briefly, $120 \mu \mathrm{l}$ MTS reagent was added to $0.6 \mathrm{ml}$ of medium in each chamber, and the cells were incubated at $37^{\circ} \mathrm{C}$ for $3 \mathrm{~h}$. Then, $120 \mu 1$ medium was transferred to a 96 -well plate, and the absorbance was detected at $490 \mathrm{~nm}$ with a Microplate Reader (VersaMax; Molecular Devices, LLC, Sunnyvale, CA, USA). The relative cell proliferation rates of the different cell groups were evaluated using the following equation: $A_{\text {treated }} / A_{\text {control }}$, where $\mathrm{A}$ is the absorbance. All of the experiments for all cell groups were repeated six times.

In vivo experimental model. The animal protocol was approved by Sir Run Run Shaw Hospital Institutional Animal Care and Use Committee (Hangzhou, China). A total of 24 male mice (athymic nu/nu; 4-6 weeks; weight, 20 g; Shanghai SLAC Laboratory Animal Co., Ltd., Shanghai, China) were used to generate a tumor model. The mice were maintained on Alpha-Dri bedding in temperature $\left(22 \pm 2^{\circ} \mathrm{C}\right)$ and humidity (30-50\%) in controlled rooms with a $12 / 12 \mathrm{~h}$ light/dark cycle. The feeding method for mice was ad libitum. A suspension of $1 \times 10^{7}$ PC-3 cells in $100 \mu$ PBS was injected subcutaneously and unilaterally into the back of each mouse to seed a prostate cancer mass. Within three weeks, the tumor masses had grown to $5-10 \mathrm{~mm}$ in diameter (Fig. 2).

RFH-enhanced chemotherapy. A total of 24 mice bearing human prostate cancer xenografts were randomly stratified into four study groups (6 mice per group) for receipt of different intratumoral treatments: i) PBS (control); ii) RFH-only $\left(42^{\circ} \mathrm{C}\right.$ for $20 \mathrm{~min}$ via the heating guidewire); iii) chemo-only (intratumoral injection of $75 \mathrm{mg} / \mathrm{m}^{2}$ docetaxel); and iv) combination therapy (chemo plus RFH). RFH was conducted by inserting the 0.022 inch heating-guidewire into the tumor such that its hot spot was at the center of the tumor. A $400 \mu \mathrm{m}$ micro-optical temperature fiber (Photon Control Inc.) was placed subcutaneously parallel to the heating guidewire, such that the real 

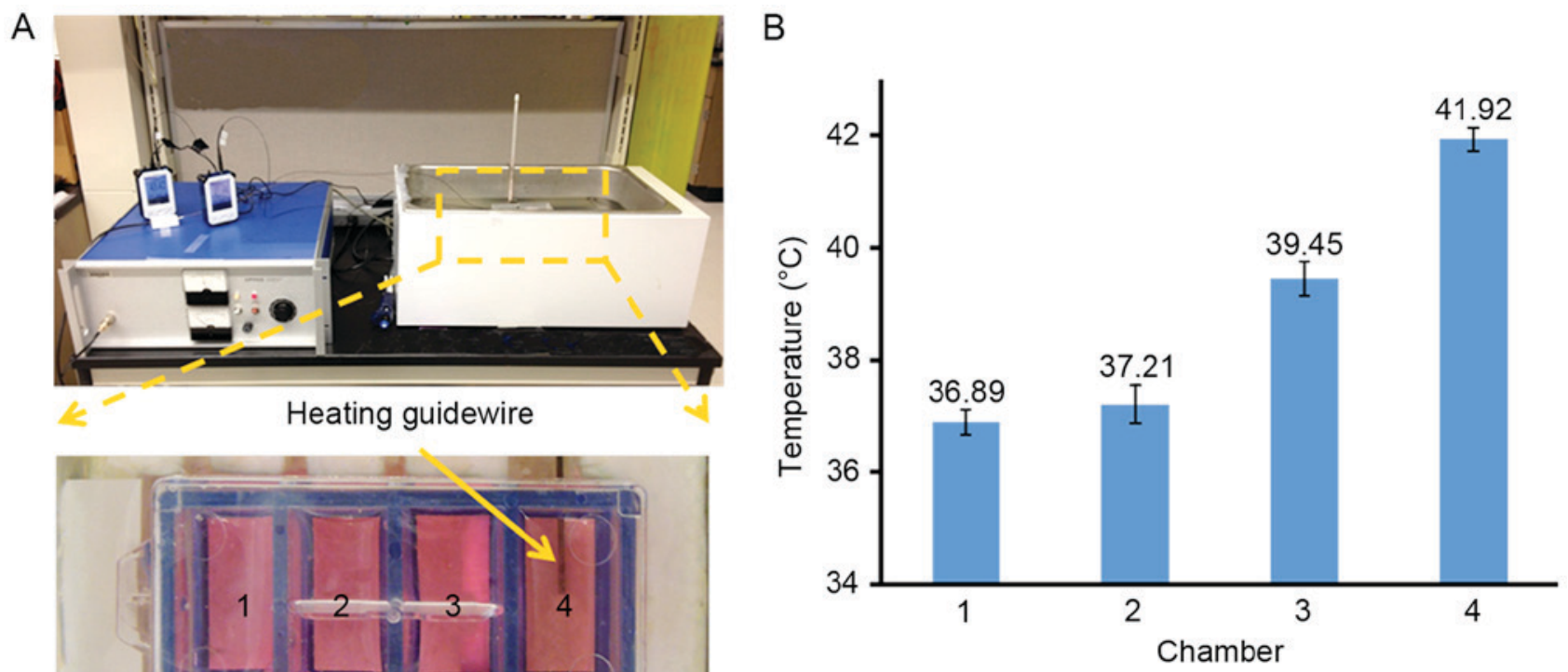

Figure 1. (A) In vitro experimental set-up for radiofrequency heating of human prostate cancer cells. The cells were seeded in a four-chamber cell culture slide, which was then placed in a water bath at $37^{\circ} \mathrm{C}$. A 0.032 inch heating guidewire (arrow) was positioned under the bottom of chamber 4 . (B) When the temperature reached $42^{\circ} \mathrm{C}$ in chamber 4 , a stable heat gradient was created along the four chambers.

A

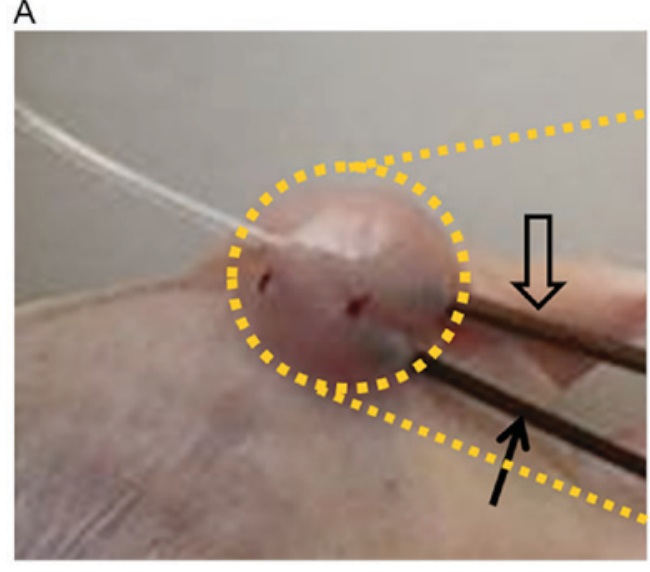

B

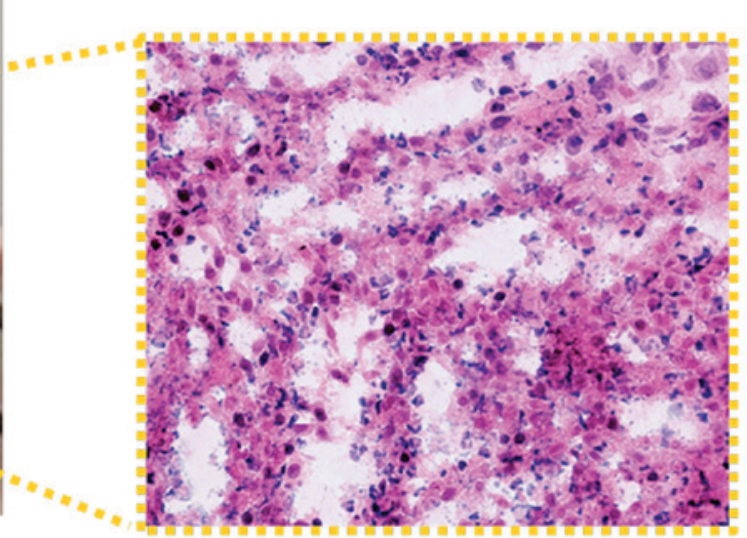

Figure 2. (A) In vivo experimental set-up for radiofrequency heat-enhanced direct intratumoral chemotherapy of a mouse with a prostate cancer xenograft (circle). Trans-tumor insertion of a 0.032 inch heating guidewire was performed (open arrow). The radiofrequency-heated tumor was maintained at $42^{\circ} \mathrm{C}$ by continuously measuring the temperature with a micro-thermometry fiber (solid arrow) placed parallel to the heating guidewire within the tumor. (B) A pathological study with hematoxylin and eosin staining confirmed the successful generation of a prostate cancer xenograft (magnification, $\mathrm{x} 400$ ).

temperature in the tumor mass was measured continuously (Fig. 2A). By adjusting the RF output power to $\sim 10 \mathrm{~W}$, the temperature of the tumor mass was maintained at $\sim 42^{\circ} \mathrm{C}$. Throughout the entire procedure, the animals were anesthetized with $1-3 \%$ isoflurane in $100 \%$ oxygen.

Magnetic resonance imaging (MRI) follow-up. The animals were placed in the supine position in a 3.0 Tesla MR scanner with a $100 \mathrm{~mm}$ micro-imaging coil (GE Healthcare, Chicago, IL, USA). MRI scans were acquired prior, and 7 and 14 days subsequent to the treatment. Axial and sagittal T2-weighted imaging was performed using a Fast Spin Echo sequence with the following parameters: TR/TE $=3,100 \mathrm{~ms} / 80 \mathrm{~ms}$, field of view $=8 \mathrm{~cm}$, matrix $=256 \times 256$, section thickness $=1.5 \mathrm{~mm}$, intersection gap $=0.5 \mathrm{~mm}$, and Number of Excitation $(\mathrm{NEX})=3$. Axial T1-weighted imaging was then achieved using a spin echo (SE) sequence with the following parameters: $\mathrm{TR} / \mathrm{TE}=550 \mathrm{~ms} / 15 \mathrm{~ms}$, field of view $=8 \mathrm{~cm}$, matrix $=256 \times 256$, section thickness $=1.5 \mathrm{~mm}$, intersection gap $=0.5 \mathrm{~mm}$, and $\mathrm{NEX}=2$.

Imaging analysis. For the tumor size measurements, the tumor volume was expressed in cubic millimeters $\left(\mathrm{mm}^{3}\right)$ and was calculated based on the axial T2-weighted images. The tumor 
border was delineated manually, and the area was automatically calculated using ImageJ 1.46r software (Wayne Rasband, National Institutes of Health, USA). Given the irregular contours of the tumors, the tumor volume was calculated according to the following equation: $\mathrm{V}=\mathrm{s} \cdot\left(\mathrm{a}_{1}+\mathrm{a}_{2}+\mathrm{a}_{3}+\ldots\right.$ $+a_{n}$ ), where $s$ is the section interval and $a_{1}-a_{n}$ are the areas of various sections.

The relative tumor volume (RTV) at different time points was then calculated for each tumor using the following equation: $\mathrm{RTV}=\mathrm{TV}_{\mathrm{Dn}} / \mathrm{TV}_{\mathrm{D} 0}$, where $\mathrm{TV}$ is the tumor volume, $D n$ is day 1,7 , or 14 following treatment and D0 is the day prior to treatment (8).

As the present study primarily focused on proving the principle of this novel concept, a longitudinal follow-up of the animals to time points at which the treated tumors had completely disappeared was not performed.

Pathological confirmation. Following satisfactory MRI, the mice were sacrificed with $5 \% \mathrm{CO}_{2}$, and the tumor masses were harvested. The tumor tissues were embedded in Tissue Freezing Medium (O.C.T compound; Thermo Fisher Scientific, Inc.), frozen in liquid nitrogen, maintained in a freezer at $-80^{\circ} \mathrm{C}$ and then sectioned into $5 \mathrm{um}$ thick slices. The tissue slices were stained with the In Situ Cell Death Detection kit and fluorescein (Roche Diagnostics, Basel, Switzerland), and were then exposed to freshly prepared proteinase $\mathrm{K}$ working solution for $15-30 \mathrm{~min}$ at $37^{\circ} \mathrm{C}(10-20 \mu \mathrm{g} / \mathrm{ml}$ in $10 \mathrm{mM}$ Tris $/ \mathrm{HCl}$;

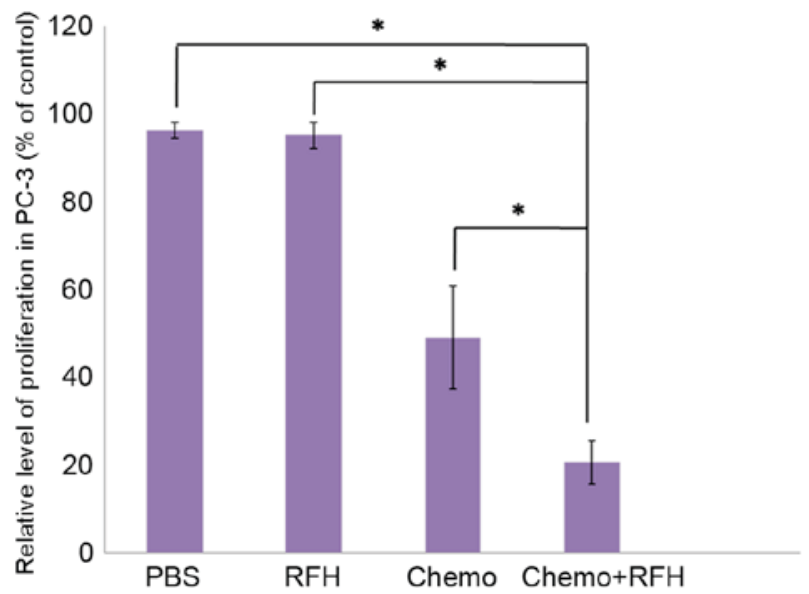

Figure 3. MTS assay demonstrating that the combination therapy (chemo + $\mathrm{RFH}$ ) exhibited a greater inhibitory effect on growth compared with RFH-only, chemotherapy-only and control group. ${ }^{*} \mathrm{P}<0.01$. RFH, radiofrequency heat.

$\mathrm{pH}$ 7.4-8). Subsequent to washing with PBS, the slices were incubated in $50 \mathrm{ml}$ TUNEL reaction mixture for $60 \mathrm{~min}$ in a dark and humidified environment. The slides were washed twice with PBS, stained overnight with DAPI (Invitrogen; Thermo Fisher Scientific, Inc.) and sealed with Prolong Gold Antifade reagent (Thermo Fisher Scientific, Inc.). All of the slides were examined under a fluorescence microscope (Leica
Week 0
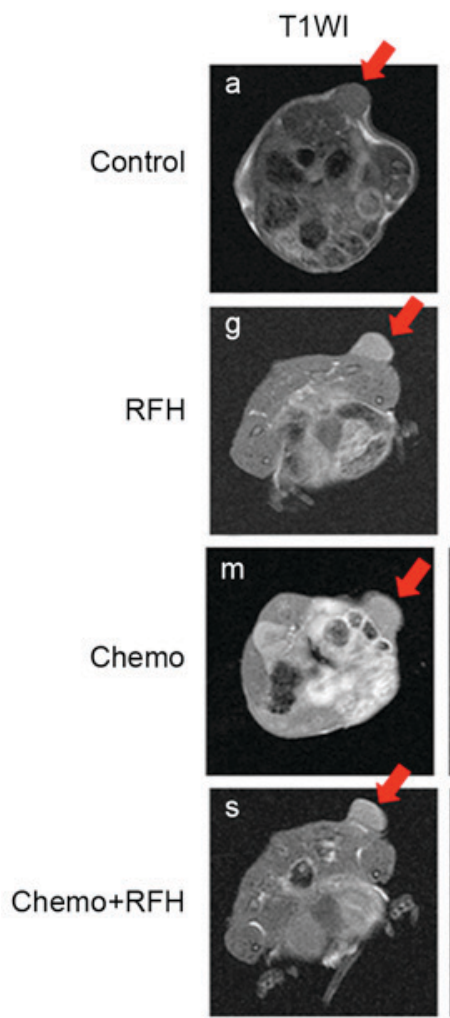

Week 1

$\mathrm{T} 2 \mathrm{WI}$
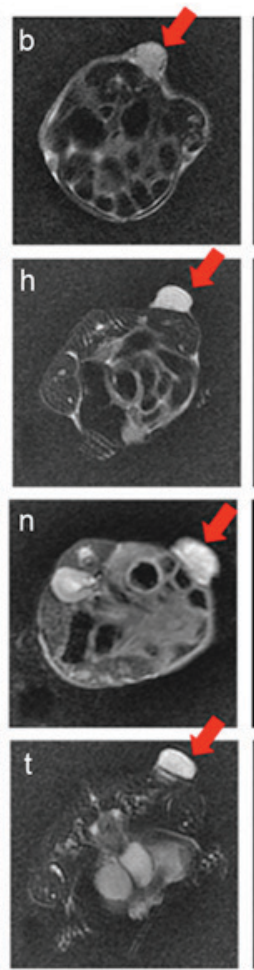

T1WI
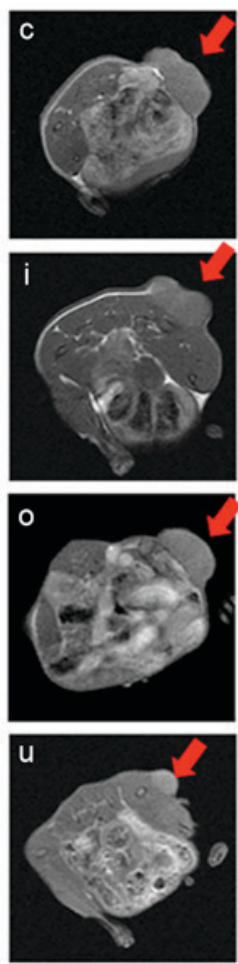

$\mathrm{T} 2 \mathrm{WI}$
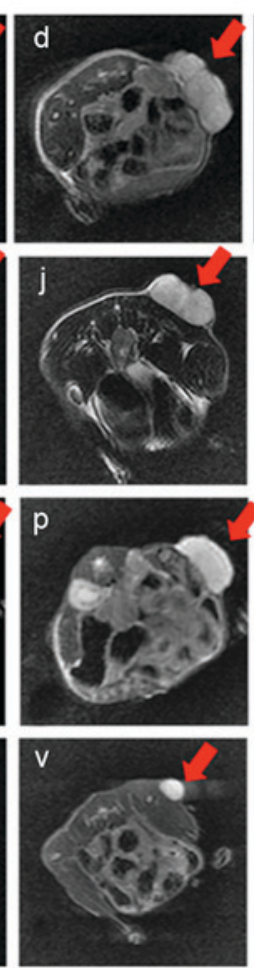

Week 2 T1WI
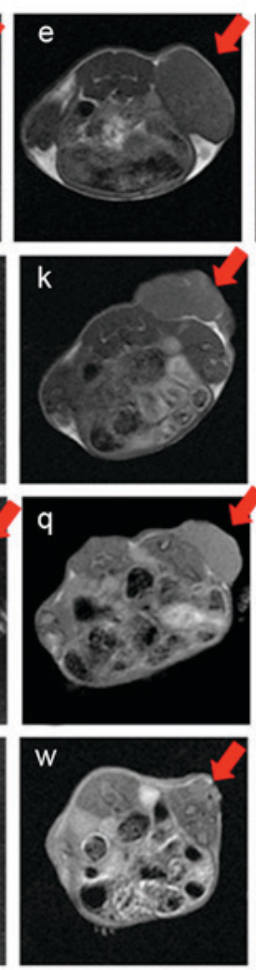

$\mathrm{T} 2 \mathrm{WI}$
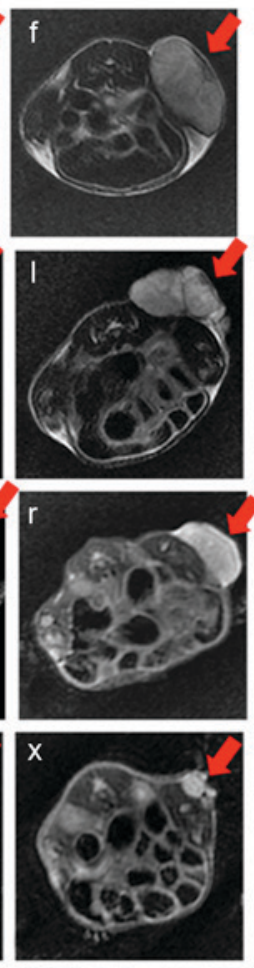

Figure 4. Representative T1-weighted images and T2-weighted images of mice bearing prostate cancer xenografts in the four treatment groups demonstrating homogeneous hypointense (T1WI) and hyperintense (T2WI) tumors (arrows) on the unilateral backs of the mice. The follow-up images of the tumors at different time points demonstrate that the tumor size in the chemo plus radiofrequency heat (RFH) group (S-X) was clearly decreased at week 2 following treatment (arrow on x) compared within the control (a-f), RFH-only (g-1), and chemo-only (m-r) groups. RFH, radiofrequency heat; T1WI, T1-weighted image; T2WI, T2-weighted image. 
Microsystems GmbH, Wetzlar, Germany), and a total of 200 cells were counted in 7 fields of view to calculate the mean percentage of apoptotic cells as the apoptotic index. Apoptotic cells were counted at $x 400$ magnification using Image-Pro Plus 6 software (Media Cybernetics, Inc., Rockville, MD, USA).

The tissue slices (5 $\mu \mathrm{m}$ in thickness) were stained with Hematoxylin and Eosin Staining kit (Nanjing SenBeiJia Biological Technology, Nanjing, China). The slides were immersed for $30 \mathrm{sec}$ and agitated by hand in $\mathrm{H}_{2} \mathrm{O}$. Slide were submerged into a Coplin jar containing Mayer's hematoxylin (Nanjing SenBeiJia Biological Technology, Nanjing, China) and agitated for $30 \mathrm{sec}$ prior to being washed in $\mathrm{H}_{2} \mathrm{O}$ for $1 \mathrm{~min}$. Slides were stained with $1 \%$ eosin Y solution (Nanjing SenBeiJia Biological Technology, Nanjing, China) for $30 \mathrm{sec}$ with agitation. Sections were then dehydrated with two changes of $95 \%$ alcohol and two changes of $100 \%$ alcohol for $30 \mathrm{sec}$ each. The alcohol was then extracted with two changes of xylene. All of the slides were examined at x400 magnification under a light microscope (Leica, Wetzlar, Germany).

Statistical analysis. Data are presented as the means \pm standard deviation. The statistical analyses were performed using SPSS 13.0 (IBM Corp., Armonk, NY, USA). One-way analysis of variance was performed to compare the mean cell proliferation rate, cell apoptotic index, tumor volume and RTV, and Tukey's post hoc test was used for multiple comparisons. $\mathrm{P}<0.05$ was considered to indicate a statistically significant difference.

\section{Results}

RFH enhanced chemotherapeutic efficiency in prostate cancer cells. In the in vitro experiments, the combination therapy significantly reduced the proliferation of prostate cancer cells compared with the control, RFH-only and chemo-only groups (Fig. 3 ; $20.50 \pm 4.99$ vs. $96.18 \pm 1.82 \%, P=0.005 ; 95.06 \pm 2.95 \%$, $\mathrm{P}=0.003 ; 48.98 \pm 11.74 \%, \mathrm{P}=0.007$, respectively).

RFH increased chemotherapeutic efficacy in a prostate cancer xenograft model. All of the animals survived throughout the in vivo experiments. A MRI analysis demonstrated significant reductions in tumor size in the mice treated with combination therapy (chemo plus RFH) compared with those that received the control, RFH-only and chemo-only treatments $\left(0.28 \pm 0.16\right.$ vs. $1.42 \pm 0.27 ; 0.96 \pm 0.23 ; 0.75 \pm 0.18 \mathrm{~cm}^{3}$, respectively; Fig. 4; $\mathrm{P}<0.001)$. These results were confirmed by subsequent pathological examination. The average RTV in the combination treatment group was significantly lower compared with the control, RFH-only and chemo-only groups $(0.57 \pm 0.17$ vs. $3.95 \pm 0.26 ; 2.68 \pm 0.43 ; 2.08 \pm 0.30$, respectively; $\mathrm{P}<0.001$; Fig. 5). The number of apoptotic cells and the average apoptotic index of the combination therapy group were significantly higher compared with the control, RFH-only and chemo-only groups (Fig. 6; $43.22 \pm 10.26$ vs. $8.00 \pm 2.87 \%, \mathrm{P}=0.003 ; 10.91 \pm 4.31 \%, \mathrm{P}=0.032 ; 23.20 \pm 6.00 \%$, $\mathrm{P}=0.025$, respectively).

\section{Discussion}

Prostate cancer is a common malignancy in men, and the worldwide burden of this disease is increasing (2). Although

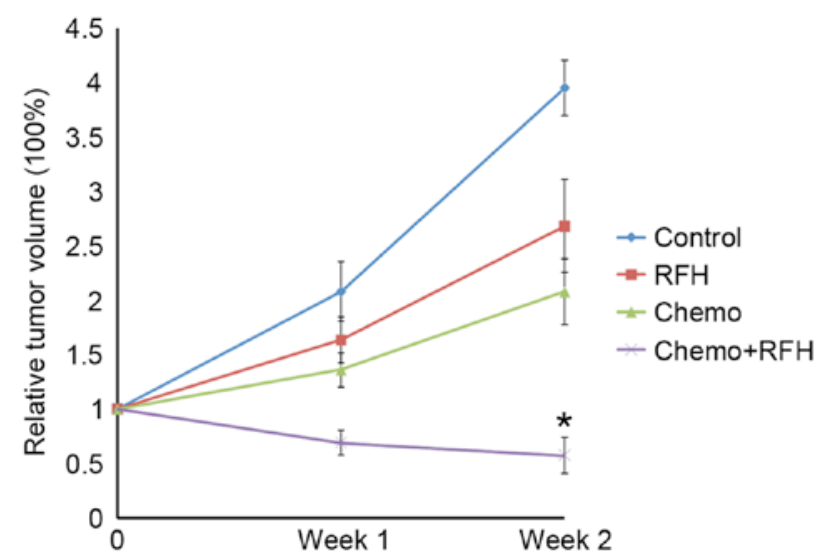

Figure 5. A comparison of the relative tumor volumes in the four treatment groups indicates that RFH-enhanced chemotherapy significantly inhibited tumor growth at week 2 post-treatment ( $\mathrm{P}<0.001)$. $\mathrm{RFH}$, radiofrequency heat.

\section{A}

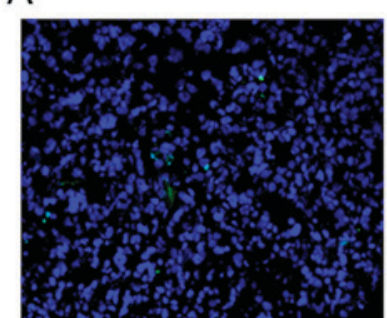

Control

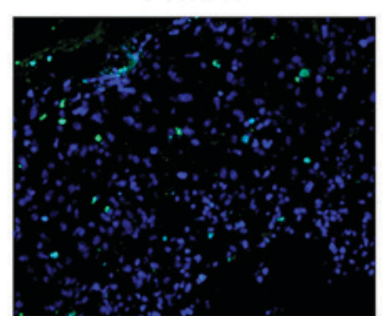

Chemo

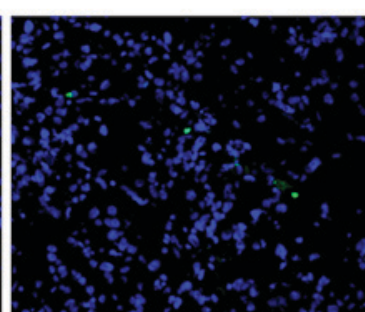

$\mathrm{RFH}$

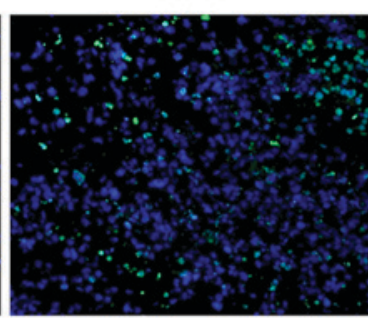

Chemo+RFH
B

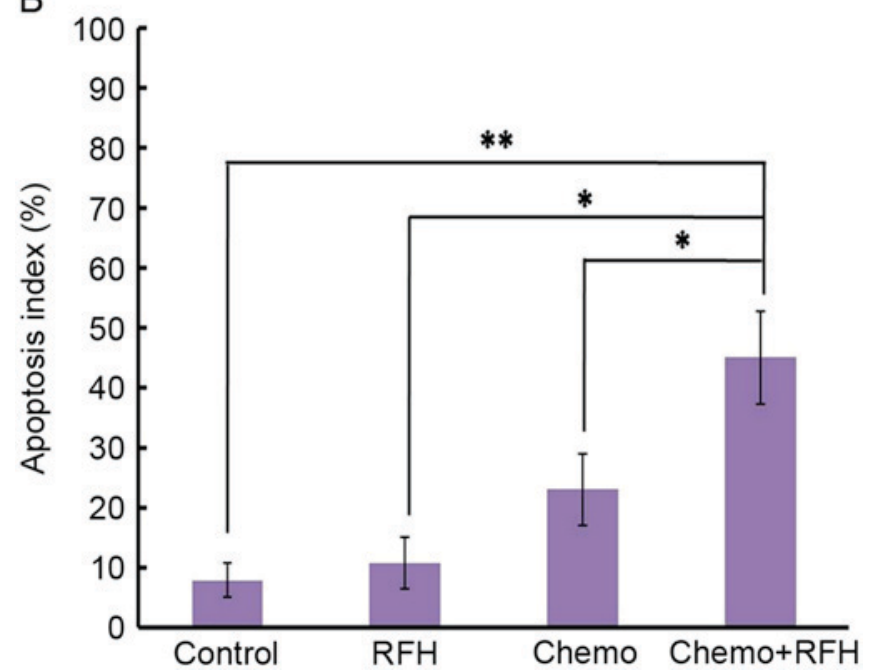

Figure 6. (A) Apoptosis assay demonstrating that there was a greater number of apoptotic cells (green) in the combination therapy group (chemo $+\mathrm{RFH}$ ) compared with the other three groups. (B) This finding was confirmed by the analysis of the apoptotic index: The apoptotic index of the combination treatment groups was higher compared with the other treatment groups. ${ }^{*} \mathrm{P}<0.05$; ${ }^{* * *} \mathrm{P}<0.01$. RFH, radiofrequency heat. 
external beam radiotherapy and whole-gland radical therapies have improved patient survival, the overtreatment of patients with low-grade and localized prostate cancer is of great concern (7). Although systemic administration of chemotherapy is a routine approach for the treatment of prostate cancer, the technology currently available does not guarantee a sufficient accumulation of chemotherapeutic agents at the target tumor site, and may cause toxicities to other vital organs (17).

Minimally invasive interventions have been demonstrated to improve the management of prostate cancer in previous studies (7). Under imaging guidance, interventional devices may be placed precisely at the target tumor site, allowing the delivery of highly concentrated therapeutics to the target cells (18). Such local approaches avoid the systemic administration of chemotherapeutic agents and therefore minimize toxicity to other organs $(19,20)$.

The present study attempted to overcome the disadvantages of the currently used systemic chemotherapy for prostate cancer by developing a novel interventional therapeutic approach that integrates the benefits of multiple technologies, namely RF technology, interventional oncology and direct intratumoral chemotherapy. Proliferation of prostate cancer cells significantly decreased in the combination therapy group compared with the control, RFH-only and chemo-only groups To the best of our knowledge, this provided the first demonstration that RFH may significantly improve the efficacy of the first-line chemotherapeutic agent docetaxel in human prostate cancer cells. These findings were consistent with previous studies by the authors (10). Zhang et al (10) demonstrated that combination therapy (chemotherapy plus RF hyperthermia) killed many more cancer cells compared with the chemotherapy-only and RF hyperthermia-only treatments in a preclinical study of cholangiocarcinoma.

Then, in the animal models with human prostate cancer xenograft in the present study, the reduction of the tumor size and RTV and the increment of the number of apoptosis cells demonstrated significant improvement using combination therapy in comparison of other groups following two weeks of treatment. Those results additionally confirmed that the direct intratumoral delivery of RFH significantly enhances the efficacy of local chemotherapy for prostate cancer. In a breast cancer study, Zhou et al (14) also reported similar efficacies of using RHF combined with doxorubicin compared to three other treatment groups: Controls, RFH-only, and chemo-only.

The present study also hypothesized that the mechanisms underlying RFH-enhanced chemotherapy may include heating to fracture the tissue, increasing the permeability of the cytoplasmic membrane, increasing cellular metabolism and increasing the activity of the heat shock protein pathway $(21,22)$. In addition, RFH may also impair the drug efflux ability of cancer cells. All of these mechanisms facilitate the entry of therapeutics into targeted tumor cells for the effective destruction of tumor tissues, and thereby improve the therapeutic outcome of prostate cancer.

Additional efforts are required to determine whether this novel combination therapy approach may increase endpoint survival by optimizing the efficacy of therapeutic regimens. In the present study, the human prostate cancer PC-3 cell line was used. As PC-3 cells are relatively fast-growing prostate cancer cells, the follow-up time was limited to two weeks following treatment. A longer follow-up period would have resulted in the xenograft tumor masses, particularly those in the control animal group, reaching $>10 \%$ of the body weight of the mice, which was not approved by the Institutional Animal Care and Use Committee. Therefore, a limitation of the present study is that it did not evaluate the long-term therapeutic effects with follow-up MRI.

In conclusion, the present study has introduced the novel concept of using interventional RFH to increase the therapeutic efficacy of local chemotherapy for prostate cancer. The present study has successfully established a foundation for the future development of a novel interventional oncology technology, namely 'interventional RFH-enhanced local chemotherapy', for the effective management of prostate cancer through the integration of RF technology, interventional oncology and direct intratumoral chemotherapy.

\section{Acknowledgements}

The present study was supported by the National Institutes of Health (grant no. R01EB012467), the National Basic Research Program of China (973 Program; grant no. 2014CB744505), the National Natural Science Foundation of China (grant nos. 81401493, 81430040, 81571738, and 81401504), and the Program for National Science and Technology Major Project of China (grant no. 2013ZX10002004-001-005).

\section{References}

1. Wolff RF, Ryder S, Bossi A, Briganti A, Crook J, Henry A, Karnes J, Potters L, de Reijke T, Stone N, et al: A systematic review of randomised controlled trials of radiotherapy for localised prostate cancer. Eur J Cancer 51: 2345-2367, 2015.

2. Cuzick J, Thorat MA, Andriole G, Brawley OW, Brown PH, Culig Z, Eeles RA, Ford LG, Hamdy FC, Holmberg L, et al: Prevention and early detection of prostate cancer. Lancet Oncol 15: e484-e492, 2014.

3. Litwin MS and Tan HJ: The diagnosis and treatment of prostate cancer: A reviwe. JAMA 317: 2532-2542, 2017.

4. Polascik TJ, Mayes JM, Sun L, Madden JF, Moul JW and Mouraviev V: Pathologic stage T2a and T2b prostate cancer in the recent prostate-specific antigen era: Implications for unilateral ablative therapy. Prostate 68: 1380-1386, 2008.

5. Stattin P, Holmberg E, Johansson JE, Holmberg L, Adolfsson J and Hugosson J; National prostate cancer register (NPCR) of Sweden: Outcomes in localized prostate cancer: National prostate cancer register of Sweden follow-up study. J Natl Cancer Inst 102: 950-958, 2010

6. Heidenreich A, Bastian PJ, Bellmunt J, Bolla M, Joniau S, van der Kwast T, Mason M, Matveev V, Wiegel T, Zattoni F, et al: EAU guidelines on prostate cancer. Part II: Treatment of advanced, relapsing, and castration-resistant prostate cancer. Eur Urol 65: 467-479, 2014.

7. Wu X, Zhang F, Chen R, Zheng W and Yang X: Recent advances in imaging-guided interventions for prostate cancers. Cancer Lett 349: 114-119, 2014.

8. Olive KP, Jacobetz MA, Davidson CJ, Gopinathan A, McIntyre D, Honess D, Madhu B, Goldgraben MA, Caldwell ME, Allard D, et al: Inhibition of Hedgehog signaling enhances delivery of chemotherapy in a mouse model of pancreatic cancer. Science 324: 1457-1461, 2009.

9. Coleman JA and Scardino PT: Targeted prostate cancer ablation: Energy options. Curr Opin Urol 23: 123-128, 2013.

10. Zhang F, Le T, Wu X, Wang H, Zhang T, Meng Y, Wei B, Soriano SS, Willis P, Kolokythas O and Yang X: Intrabiliary RF heat-enhanced local chemotherapy of a cholangiocarcinoma cell line: Monitoring with dual-modality imaging-preclinical study. Radiology 270: 400-408, 2014. 
11. Zhang T, Zhang F, Meng Y, Wang H, Le T, Wei B, Lee D, Willis $\mathrm{P}$, Shen $B$ and Yang X: Diffusion-weighted MRI monitoring of pancreatic cancer response to radiofrequency heat-enhanced intratumor chemotherapy. NMR Biomed 26: 1762-1267, 2013.

12. Owusu RA, Abern MR and Inman BA: Hyperthermia as adjunct to intravesical chemotherapy for bladder cancer. Biomed Res Int 2013: 262313, 2013.

13. Serpa Neto A, Tobias-Machado M, Kaliks R, Wroclawski ML, Pompeo AC and Del Giglio A: Ten years of docetaxel-based therapies in prostate adenocarcinoma: A systematic review and meta-analysis of 2244 patients in 12 randomized cliniical trials. Clin Genitourin Cancer 9: 115-123, 2011.

14. Zhou Y, Han G, Wang Y, Hu X, Li Z, Chen L, Bai W, Luo J, Zhang Y, Sun J and Yang X: Radiofrequency heat-enhanced chemotherapy for breast cancer: Towards interventional molecular image-guided chemotherapy. Theranostics 4: 1145-1152, 2014.

15. Morikawa Y, Koike H, Sekine Y, Matsui H, Shibata Y, Ito K and Suzuki K: Rapamycin enhances docetaxel-induced cytotoxicity in a androgen-independent prostate cancer xenograft model by survivin downregulation. Biochem Biophys Res Commun 419: 584-589, 2012.

16. Williams JF, Muenchen HJ, Kamradt JM, Korenchuk S and Pienta KJ: Treatment of androgen-independent prostate cancer using antimicrotubule agents docetaxel and estramustine in combination: An experimental study. Prostate 44: 275-278, 2000.
17. Kellokumpu-Lehtinen PL, Hjälm-Eriksson M, Thellenberg-Karlsson C, Åström L, Franzen L, Marttila T, Seke M, Taalikka M and Ginman C; SPCG-13: Toxicity in patients receiving adjuvant docetaxel + hormonal treatment after radical radiotherapy for intermediate or high-risk prostate cancer: A preplanned safety report of the SPCG-13 trial. Prostate Cancer Prostatic Dis 15: 303-307, 2012.

18. Johannsen M, Thiesen B, Wust P and Jordan A: Magnetic nanoparticle hyperthermia for prostate cancer. Int J Hyperthemia 26: 790-795, 2010.

19. Kim KW, Chow O, Parikh K, Blank S, Jibara G, Kadri H, Labow DM and Hiotis SP: Peritoneal carcinomatosis in patients with gastric cancer, and the role for surgical resection, cytoreductive surgery, and hyperthermic intraperitoneal chemotherapy. Am J Surg 207: 78-83, 2014.

20. Ahmed M, Brace CL, Lee FT Jr and Goldberg SN: Principles of and advances in percutaneous ablation. Radiology 258: 351-369, 2011.

21. Madio DP, van Gelderen P, DesPres D, Olson AW, de Zwart JA, Fawcett TW, Holbrook NJ, Mandel M and Moonen CT: On the feasibility of MRI-guided focused ultrasound for local induction of gene expression. J Magn Reson Imaging 8: 101-104, 1998.

22. Wei Z, Ye X, Yang X, Zheng A, Huang G, Li W, Ni X, Wang J and Han X: Microwave ablation in combination with chemotherapy for the treatment of advanced non-small cell lung cancer. Cardiovasc Intervent Radiol 38: 135-142, 2015. 